\title{
Saúde mental de adolescentes em tempos de Covid-19: desafios e possibilidades de enfrentamento
}

\author{
| ${ }^{1}$ Claudia Reis Miliauskas, ${ }^{2}$ Daniela Porto Faus |
}

\author{
1 Psicologia Médica, Universidade do Estado do Rio de Janeiro. Rio de Janeiro-RJ, Brasil (claudiarmili@gmail.com). \\ ORCID: 0000-0002-9077-5170 \\ ${ }_{2}$ Maternidade-Escola, Universidade Federal do Rio de Janeiro. Rio de Janeiro-RJ, Brasil (daniela.faus@me.ufrj.br). \\ ORCID: 0000-0002-4952-7327 \\ Recebido em: 25/06/2020 \\ Aprovado em: 15/07/2020 \\ Revisado em: 18/09/2020
}

DOI: http://dx.doi.org/10.1590/S0103-73312020300402

A crise sanitária motivada pelo novo coronavírus (SARS-CoV-2), causador da doença Covid-19, tem afetado a vida de grande parte da população mundial (OPAS-BRASIL, 2020; WHO, 2020a, b). Diante da falta de vacina ou tratamento adequado, a OMS tem proposto, como principal forma de combate à pandemia, distanciamento social (HELLEWELL et al., 2020). As apresentaçōes mais graves da doença têm sido observadas principalmente em idosos e pessoas com doenças crônicas (WU; McGOOGAN, 2020). No entanto, as medidas de isolamento social vêm impactando um espectro maior da populaçáo, sendo os adolescentes especialmente vulneráveis ao adoecimento mental neste contexto, devido à importância dos pares e do convívio em grupo para essa faixa etária (BOHOSLAVSKY, 2007; BOWEN; WALKER, 2015; LEVY, 2013).

Passados os primeiros meses da pandemia, é possível identificar estudos que apontam para o aumento de depressão, estresse e ansiedade (FILGUEIRAS; STULTS-KOLEHMAINEN, 2020; VINDEGAARD; BENROS, 2020; ZHANG; ZHANG; MA; DI, 2020), alteração na qualidade do sono (ZHANG; ZHANG; MA; DI, 2020), uso de substâncias psicotrópicas (FEGERT et al., 2020), dentre 
outros efeitos deletérios à saúde nesse período. Além destas evidências empíricas referentes à pandemia da Covid-19, é possível conjecturar as consequências negativas à saúde mental que o isolamento pode gerar a partir de experiências anteriores. Após as epidemias de Ebola e SARS-Cov, foram reportados aumentos de ansiedade e estresse pós-traumático em profissionais de saúde (TORALES et al., 2020). Além disso, observou-se o aumento de marcadores biológicos relacionados ao estresse e sintomas neurológicos funcionais durante o surto de MERS-Cov e após o distanciamento social instituído devido ao atentado terrorista da Maratona de Boston (TORALES et al., 2020). Quando se foca especificamente nas consequências do isolamento social para os adolescentes, as pesquisas são mais escassas; entretanto, uma revisão sistemática destacou o aumento da depressão e da ansiedade neste grupo (LOADES et al., 2020).

\section{Especificidades da adolescência que aumentam a vulnerabilidade para o adoecimento emocional}

A adolescência pode ser entendida como a fase de desenvolvimento que ocorre entre a infância e a vida adulta. Este é um momento de consideráveis mudanças físicas, psicológicas, cognitivas e socioculturais, sendo considerado, por alguns autores, como período esperado de crise (CARTER; MCGOLDRICK, 2001). O processo de individuação, que ocorre desde o nascimento até o falecimento, se intensifica no período da adolescência. Com isso, são esperados o questionamento dos valores provenientes da família de origem e a busca por outras referências. Esse movimento se dá normalmente através dos pares, sendo importantíssimas nesse período as relaçôes sociais de amizade (BOHOSLAVSKY, 2007; LEVY, 2013). É comum o adolescente náo se separar dos amigos mais próximos, se afetando de forma profunda pelos valores e julgamentos dos colegas. Esse processo é chamado de uniformidade e traz segurança ao adolescente (BRASIL, 2017). Em paralelo, se constitui como o momento no qual o indivíduo passa a ter mais responsabilidades escolares e profissionais e aumenta o tempo fora de casa.

Dentre as medidas de distanciamento social, destaca-se, para este grupo, o fechamento de escolas, clubes, academias, shoppings, praias e parques. Com isso, passam a ficar restritos ao ambiente doméstico, sem a possibilidade de se relacionar fisicamente com seus pares e, possivelmente, aumentando a procura por jogos virtuais, acesso a vídeos e uso de redes sociais (BALHARA et al., 2020). Adicionado 
a isso, impôs-se o excesso de contato dentro do núcleo familiar e, porventura, ausência de privacidade. Enquanto há relato das famílias com crianças de que estas têm demonstrado felicidade frente ao aumento do tempo com os pais, essa não é a realidade mais comum para os adolescentes, que vivenciam uma interrupção no processo de busca por identidade fora de casa. O distanciamento físico atual também é acompanhado de impactos econômicos para algumas famílias, o que pode aumentar o conflito dentro das casas e a insegurança emocional dos adolescentes (MARQUES et al., 2020).

\section{Possíveis intervençóes para o enfrentamento do adoecimento mental na pandemia}

Já existe, ainda de forma incipiente, um conhecimento dos fatores que favorecem o adoecimento destes adolescentes em isolamento social. Exposição excessiva às informaçôes (GAO et al., 2020), diminuição da atividade física (STANTON et al., 2020; ZHANG et al., 2020), alteraçáo da dieta e do padrão do sono (STANTON et al., 2020) e o consumo de álcool e tabaco (STANTON et al., 2020) são alguns dos comportamentos que parecem estar relacionados com o aumento da vulnerabilidade dos jovens na pandemia. Além disso, o tempo de isolamento parece ser importante preditor de futuros problemas em saúde mental, mais, inclusive, que a intensidade do distanciamento social (LOADES et al., 2020). Diante dos desafios apresentados, é importante que se construa uma mobilização no sentido de prevenir, identificar, acolher, encaminhar e tratar problemas relacionados à saúde mental nos adolescentes. Para colaborar com essa discussão, a partir das evidências sobre o adoecimento emocional de jovens, tanto no período da pandemia quanto fora dele, elencamos algumas possibilidades de ação.

Primeiramente, podemos destacar a importância da família em auxiliar o adolescente a passar por este período com menor sofrimento e prejuízo. Exemplos de açôes de cuidado incluem o diálogo aberto sobre a pandemia, incluindo fontes de informações adequadas. Como mencionado anteriormente, o excesso de informação já se mostrou fator de risco para ansiedade (GAO; ZHENG; JIA; CHEN et al., 2020); porém a falta desta ou a presença de informaçôes falsas podem também contribuir para o aumento do estresse. Com o distanciamento social, a divisão das tarefas domésticas e algumas regras da casa precisarão ser reformuladas. É importante 
que todos os membros da família participem destes novos combinados, de forma a poder contribuir para a construção de um convívio familiar mais saudável. Manter um ambiente de privacidade pode ser um desafio para algumas famílias, mas o esforço neste sentido é importante de se construir. Pensar junto com o adolescente formas de manutenção do contato com amigos e familiares, ainda que no formato virtual, também auxilia a promoção da saúde mental (KIRÁLY et al., 2020).

No que tange à rotina escolar, os pais podem contribuir dialogando com os filhos sobre expectativas e dificuldades frente a este momento, auxiliando na organização da rotina e elaborando um plano de estudo. Podem, também, estar atentos às outras atividades fundamentais para o aprendizado como exercício físico ou hobbies. Os familiares devem estar sensibilizados à percepção de mudanças no comportamento do adolescente a fim de buscar ajuda de profissionais de saúde mental para orientação e tratamento, evitando a cronicidade dos sintomas e maiores consequências. Diante dos efeitos deletérios à saúde mental, se faz relevante que a família fique atenta às fases de relaxamento do distanciamento social. É possível que adolescentes apresentem comportamentos inesperados como dificuldade para retornar a algumas atividades. Por isso, frente a cada etapa, pais e filhos devem combinar juntos o que cabe para aquela família, sendo de fundamental relevância que pais funcionem como modelos neste processo (PRIME; BROWNE; WADE, 2020).

Nas possibilidades de atuação relacionadas à educação, tanto professores quanto estudantes foram forçados a se adaptar ao formato virtual. Por isso, é preciso estar atento às possibilidades individuais de cada adolescente, assim como de cada núcleo familiar. Além de aspectos objetivos, acesso a computador e internet, local adequado para o estudo e sobrecarga doméstica, destacam-se as questóes subjetivas, como motivação e interesse pelas atividades, que podem dificultar o aprendizado ou favorecer o adoecimento mental. Cabe aos profissionais da educação pensar a readequação do conteúdo escolar, priorizando a saúde emocional e reformulando as formas de aprendizado conforme a evolução das fases da pandemia (SANTOS, 2020). Algumas açóes em nível mais amplo poderiam ser promovidas pelo Estado. Destacam-se a capacitação de professores para o ensino remoto emergencial, reavaliaçáo do calendário escolar com possível adiamento de exames nacionais, mapeamento das necessidades tecnológicas e digitais dos estudantes e professores, amenização das desigualdades de acesso a estas tecnologias. 
Açôes relacionadas aos serviços de saúde também são fundamentais para tratamento de adolescentes com sofrimento psíquico antes da pandemia, evitando novas crises, assim como para aqueles que podem vir a adoecer. Exemplos de intervenções para este período incluem a realização de grupos virtuais; acompanhamento individual de forma remota (CRUZ et al., 2020); seguimento das orientações de segurança sanitária e avalição da equipe para acompanhamentos presencias, quando necessários (GOVERNO DO ESTADO DO ESPÍRITO SANTO, 2020); manutençâo do contato por telefone ou vídeo com adolescente e família (CRUZ et al., 2020); flexibilização da consulta presencial para disponibilização de receitas médicas para quem já faz uso de medicações, além de atividades de telematriciamento entre equipes de saúde mental e atenção primária (MARTÍNEZ et al., 2018).

A arte, a cultura e o esporte também têm papel crucial na promoção da saúde mental da população como um todo, mas especialmente dentre os adolescentes. A produção de conteúdo que favoreça a imaginação e a criatividade, que fortaleça a percepção do adolescente de pertencimento a grupos ou tribos pode favorecer o seu desenvolvimento saudável neste momento. Além disso, campanhas específicas voltadas a esse público com temas como formas de enfrentamento do distanciamento social, estratégias para a manutenção da saúde física e mental, identificação de sinais de alerta e orientação de que serviços estão disponíveis para atendimento podem auxiliar as famílias a lidar com as dificuldades do momento e a buscar orientação profissional em caso de maior necessidade (KIRÁLY et al., 2020; LATGÉ; ARAUJO; JUNIOR, 2020).

\section{Considerações finais}

É muito importante que população e governos se alinhem no sentido de reduzir a propagação da Covid-19. Até o momento, o distanciamento social segue sendo a principal forma de redução da velocidade de transmissão da doença. Entretanto, a partir das evidências acumuladas até agora, não se devem ignorar as consequências deletérias do isolamento social à população, dentre estas o adoecimento mental de adolescentes. Familiares, educadores, profissionais de saúde, artistas e governantes podem contribuir de forma ativa no enfrentamento do adoecimento mental de adolescentes, enfatizando que distanciamento social deve ser físico, e não emocional. ${ }^{1}$ 


\section{Referências}

BALHARA, Y. P. S. et al. Impact of Lockdown Following COVID-19 on the Gaming Behavior of College Students. Indian Journal of Public Health, v. 64, p. S172-176, 2020.

BOHOSLAVSKY, R. Orientação Vocacional a estratégia clinica. São Paulo: Martins Fontes, 2007. $218 \mathrm{p}$.

BOWEN, E.; WALKER, K. Contextualising Violence and Abuse in Adolescent Romantic Relationships In: BOWEN, E.; WALKER, K. The Psychology of Violence in Adolescent Romantic Relationship. New York: Palgrave Macmillan, 2015. p. 191-191.

BRASIL. Minisério da Saúde. Proteger e Cuidar da Saúde de Adolescentes na Atençâo Básica. 1 ed. Brasília: MS, 2017.

CARTER, B.; MCGOLDRICK, M. As Mudanças no Ciclo de Vida Familiar: uma estrutura para a terapia familiar. Porto Alegre: Artmed, 2001.

CRUZ, N. M. L. V. et al. Apoio psicossocial em tempos de COVID-19: experiências de novas estratégias de gestão e ajuda mútua no sul da Bahia, Brasil. APS em Saúde, v. 2, n. 2, p. 97-105, 2020.

FEGERT, J. M. et al. Challenges and burden of the Coronavirus 2019 (COVID-19) pandemic for child and adolescent mental health: a narrative review to highlight clinical and research needs in the acute phase and the long return to normality. Child and Adolescent Psychiatry and Mental Health, v. 14, n. 1, p. 20, 2020/05/12 2020.

FILGUEIRAS, A.; STULTS-KOLEHMAINEN, M. Factors linked to changes in mental health outcomes among Brazilians in quarantine due to COVID-19. MedRxiv (preprint), 2020. GAO, J. et al. Mental health problems and social media exposure during Covid-19 outbreak. PLOS ONE, v. 15, n. 4, p. e0231924, 2020.

GOVERNO DO ESTADO DE ESPÍRITO SANTO. Nota Técnica Covid-19 no 048/2020. SESA/SSAS/GROSS/NEAE - Recomendações à Rede de Atenção Psicossocial sobre estratégias de organizaçãonno contexto da infecção da Covid-19 causada pelo novo coronavírus (SARSCoV2), orientando que os pontos de Atenção da RAPS, gestores e profissionais adotem procedimentos quanto ao funcionamento. Vitória, 2020.

HELLEWELL, J. et al. Feasibility of controlling COVID-19 outbreaks by isolation of cases and contacts. Lancet Glob Health, 8, p. e488-496, 2020.

KIRÁLY, O. et al. Preventing problematic internet use during the COVID-19 pandemic: Consensus guidance. Comprehensive Psychiatry, 100, p. 152180, 2020/07/01/ 2020.

LATGÉ, P. K.; ARAUJO, D. N.; JUNIOR, A. G. d. S. Comunicação, educação e vigilância popular em saúde em tempos de COVID-19 - a experiência das comunidades de Niterói, RJ. APS em Saúde, v. 2, n. 2, p. 122-127, 2020. 
LEVY, R. O adolescente. In: EIZIRIK, C. L.; BASSOLS, A. M. S. (Ed.). O Ciclo da Vida Humana: uma perspectiva psicodinâmica. 2 ed. Porto Alegre: Artmed, 2013. p.167-179.

LOADES, M. E. et al. Rapid Systematic Review: The Impact of Social Isolation and Loneliness on the Mental Health of Children and Adolescents in the Context of Covid-19. Journal of the American Academy of Child \& Adolescent Psychiatry, 03 jun. 2020.

MARQUES, E. S. et al. A violência contra mulheres, crianças e adolescentes em tempos de pandemia pela Covid-19: panorama, motivaçôes e formas de enfrentamento. Cadernos de Saúde Pública, v. 36, n. 4, 2020.

MARTÍnEZ, V. et al. Remote Collaborative Depression Care Program for Adolescents in Araucanía Region, Chile: Randomized Controlled Trial. Journal of Medical Internet Research, 20, n. 1, p. e38, 2018.

ORGANIZAÇÃO PANAMERICANA DA SAÚDE. Folha informativa - Covid-19 (doença causada pelo novo coronavirus). Brasília, 2020. Disponível em: https://www.paho.org/bra/ index.php?option=com_content $\&$ view $=$ article $\& i d=6101$ : $\operatorname{covid} 19 \&$ Itemid $=875$. Acesso em: 7 ago. 2020.

PRIME, H.; BROWNE, D. T.; WADE, M. Risk and Resilience in Family Well-Being During the Covid-19 Pandemic. American Psychologist, v. 75, n. 5, p. 631-643, 2020.

SANTOS, H. M. R. d. Os desafios de educar através da Zoom em contexto de pandemia: investigando as experiências e perspetivas dos docentes portugueses. Práxis Educativa, v. 15, n. e2015805, p. 1-17, 2020.

STANTON R. et al. Depression, Anxiety and Stress during Covid-19: Associations with Changes in Physical Activity, Sleep, Tobacco and Alcohol Use in Australian Adults. Int. J. Environ. Res. Public Health, v. 17, n. 11, p. 4065, 2020.

TORALES, J. et al. The outbreak of Covid-19 coronavirus and its impact on global mental health. International Journal of Social Psychiatry, v. 66, n. 4, p. 317-320, 2020/06/01 2020.

VINDEGAARD, N.; BENROS, M. E. Covid-19 pandemic and mental health consequences: Systematic review of the current evidence. Brain, behavior, and immunity, p. S08891591(0820)30954-30955, 2020.

WORLD HEALTH ORGANIZATION. WHO announces Covid-19 outbreak a pandemic. 2020a. Disponível em: https:/www.euro.who.int/en/health-topics/health-emergencies/ coronavirus-covid-19/news/news/2020/3/who-announces-covid-19-outbreak-a-pandemic. Acesso em: 7 ago. 2020.

. WHO Director-General's opening remarks at the media briefing on Covid-19. Geneva, 11 March 2020. 2020b. Acesso em: 7 ago. 2020. 
WU, Z.; MCGOOGAN, J. M. Characteristics of and Important Lessons From the Coronavirus Disease 2019 (COVID-19) Outbreak in China: Summary of a Report of 72314 Cases From the Chinese Center for Disease Control and Prevention. JAMA, v. 323, n. 13, p. 1239-1242, 2020. ZHANG, Y. et al. Mental Health Problems during the Covid-19 Pandemics and the Mitigation Effects of Exercise: A Longitudinal Study of College Students in China. International journal of environmental research and public health, v. 17, n. 10, p. 3722, 2020.

\section{Nota}

${ }^{1}$ C. R. Miliauskas e D. P. Faus foram responsáveis pela revisão bibliográfica, redação e revisão do texto. 